\title{
A Self-Improvement to the Cauchy-Schwarz Inequality
}

\author{
Stephen G Walker \\ Department of Mathematics, University of Texas at Austin, Texas, U. S. A. Email: \\ s.g.walker@math.utexas.edu
}

\section{Abstract}

We present a self improvement to the Cauchy-Schwarz inequality, which in the probability case yields

$$
[E(X Y)]^{2} \leq E\left(X^{2}\right) E\left(Y^{2}\right)-(|E(X)| \sqrt{\operatorname{Var}(Y)}-|E(Y)| \sqrt{\operatorname{Var}(X)})^{2} .
$$

It is to be noted that the additional term to the inequality only involves the marginal first two moments for $X$ and $Y$, and not any joint property. We also provide the discrete improvement to the inequality.

Keywords: Cauchy-Schwarz; Cramer-Rao Inequality, Wasserstein distance

\section{Introduction}

The Cauchy-Schwarz inequality is arguably one of the most widely used inequalities in mathematics; see [6]. Let $x=\left(x_{1}, \ldots, x_{n}\right)$ and $y=\left(y_{1}, \ldots, y_{n}\right)$ be two real sequences; then

$$
\left(\sum_{i=1}^{n} x_{i} y_{i}\right)^{2} \leq \sum_{i=1}^{n} x_{i}^{2} \sum_{i=1}^{n} y_{i}^{2}
$$

with equality if and only if there exists a constant $c$ such that $x_{i}=c y_{i}$ for all $i=1, \ldots, n$. There are a number of proofs available in the literature and textbooks.

There are refinements of (1), such as [2], but are quite specific in the details on the $x$ and $y$. A survey of the inequality is given in [4] and a recent generalization is presented in [7].

Preprint submitted to Elsevier

October 26, 2016

(C) 2016. This manuscript version is made available under the Elsevier user license http://www.elsevier.com/open-access/userlicense/1.0/ 
The aim in this paper is to provide a new and elementary (self) improvement of the Cauchy-Schwarz inequality which only uses the terms

$$
\sum_{i=1}^{n} x_{i}, \quad \sum_{i=1}^{n} x_{i}^{2}, \quad \sum_{i=1}^{n} y_{i}, \quad \text { and } \quad \sum_{i=1}^{n} y_{i}^{2} .
$$

These are the first two marginal moments for $x$ and $y$. The self improvement is similar in spirit to one appearing in [8], which worked on the Jensen inequality, and also in [3], which worked on the logarithmic Sobolev inequality.

Other improvments of the Cauchy-Schwarz inequality use more than these first two moments; see for example [1], which use joint properties of $x$ and $y$.

In the following we will use $\bar{x}$ and $\bar{y}$ to denote $n^{-1} \sum_{i=1}^{n} x_{i}$ and $n^{-1} \sum_{i=1}^{n} y_{i}$, respectively, and $V_{x}$ and $V_{y}$ to denote

$$
\sum_{i=1}^{n} x_{i}^{2}-n \bar{x}^{2} \text { and } \sum_{i=1}^{n} y_{i}^{2}-n \bar{y}^{2}
$$

respectively. It is clear that $V_{x}$ and $V_{y}$ are nonnegative. The new result is that

$$
\left(\sum_{i=1}^{n} x_{i} y_{i}\right)^{2} \leq \sum_{i=1}^{n} x_{i}^{2} \sum_{i=1}^{n} y_{i}^{2}-n\left(|\bar{x}| \sqrt{V_{y}}-|\bar{y}| \sqrt{V_{x}}\right)^{2} .
$$

We prove this in section 2 where we also present the improved inequality for the probability setting. Section 3 contains some applications.

\section{Proof of (2)}

Now (2) is a self-improvement of the Cauchy-Schwarz inequality. To see this, for some as yet unspecified $h_{1}$ and $h_{2}$, we have

$$
\left(\sum_{i=1}^{n}\left(x_{i}-h_{1}\right)\left(y_{i}-h_{2}\right)\right)^{2} \leq \sum_{i=1}^{n}\left(x_{i}-h_{1}\right)^{2} \sum_{i=1}^{n}\left(y_{i}-h_{2}\right)^{2}
$$

SO

$$
\left(\sum_{i=1}^{n} x_{i} y_{i}-h_{1} n \bar{y}-h_{2} n \bar{x}+n h_{1} h_{2}\right)^{2} \leq\left(\sum_{i=1}^{n} x_{i}^{2}-2 h_{1} n \bar{x}+n h_{1}^{2}\right)\left(\sum_{i=1}^{n} y_{i}^{2}-2 h_{2} n \bar{y}+n h_{2}^{2}\right) .
$$


Setting $h_{1} h_{2}=h_{1} \bar{y}+h_{2} \bar{x}$, to isolate the $\sum_{i=1}^{n} x_{i} y_{i}$ term from the left side, and after some algebra on the right side, we arrive at

$$
\left(\sum_{i=1}^{n} x_{i} y_{i}\right)^{2} \leq \sum_{i=1}^{n} x_{i}^{2} \sum_{i=1}^{n} y_{i}^{2}-n h_{1} V_{y}\left[2 \bar{x}-h_{1}\right]-n h_{2} V_{x}\left[2 \bar{y}-h_{2}\right]
$$

Now setting $h_{1}=h$ and $h_{2}=h \bar{y} /(h-\bar{x})$ for some $h$, we have

$$
h_{1} V_{y}\left[2 \bar{x}-h_{1}\right]+h_{2} V_{x}\left[2 \bar{y}-h_{2}\right]=h(2 \bar{x}-h)\left[V_{y}-V_{x} \frac{\bar{y}^{2}}{(h-\bar{x})^{2}}\right] \text {. }
$$

Putting $h=\bar{x}+t$ the aim now would be to maximize,

$$
F(t)=\left(\bar{x}^{2}-t^{2}\right)\left(V_{y}-V_{x} \bar{y}^{2} / t^{2}\right) .
$$

Noting that $F(\infty)=-\infty$ and $F(0+)=-\infty$, we see the maximizing $t^{2}$ is given by $\widehat{t^{2}}=|\bar{x} \bar{y}| \sqrt{V_{x} / V_{y}}$, and so (3) becomes

$$
\left(|\bar{x}| \sqrt{V_{y}}-|\bar{y}| \sqrt{V_{x}}\right)^{2}
$$

This completes the proof.

In the special case when $x_{i}=1$ for all $i$, we obtain an equality in (2).

Corollary. It is that

$$
\frac{1}{2} \sum_{i=1}^{n} \sum_{j=1}^{n}\left(x_{i} y_{j}-x_{j} y_{i}\right)^{2} \geq n\left(|\bar{x}| \sqrt{V_{y}}-|\bar{y}| \sqrt{V_{x}}\right)^{2} .
$$

The Lagrange identity (see [6]) has

$$
\sum_{i=1}^{n} x_{i}^{2} \sum_{i=1}^{n} y_{i}^{2}-\left(\sum_{i=1}^{n} x_{i} y_{i}\right)^{2}=\frac{1}{2} \sum_{i=1}^{n} \sum_{j=1}^{n}\left(x_{i} y_{j}-x_{j} y_{i}\right)^{2}
$$

and hence the statement of the Corollary follows.

The probability version of the Cauchy-Schwarz inequality is given by

$$
[E(X Y)]^{2} \leq E\left(X^{2}\right) E\left(Y^{2}\right)
$$


and is an equality if and only if $Y=c X$ for some constant $c$.

The self improvement for this inequality follows the same outline as in th proof of (2) and we omit the details. The new inequality is given by

$$
[E(X Y)]^{2} \leq E\left(X^{2}\right) E\left(Y^{2}\right)-(|E(X)| \sqrt{\operatorname{Var}(Y)}-|E(Y)| \sqrt{\operatorname{Var}(X)})^{2} .
$$

\section{Applications}

\subsection{Standard normal}

We obtain an interesting result when we take $Y$ to be standard normal; then (5) becomes

$$
[E(X Y)]^{2} \leq \operatorname{Var}(X)
$$

Of course, this would also hold if $Y$ had mean 0 and variance 1 .

To understand this inequality better, let us consider the Wasserstein distance with quadratic cost function; i.e.

$$
d(X, Y)=\inf _{\pi \in C(p)} \iint(x-y)^{2} \pi(\mathrm{d} x, \mathrm{~d} y)
$$

where $C(p)$ is the set of probability measures $\pi(x, y)$ which have the standard normal as marginal for $Y$ and the marginal $p(x)$ for $X$. See, for example, [5]. It is well known that the minimum is obtained when $\pi(x, y)$ has $X$ from $p(x)$ with

$$
Y=\Phi^{-1}(P(X))
$$

where $P(x)$ is the distribution function corresponding to the density $p(x)$ and $\Phi$ is the standard normal distribution function. Hence, since the marginals of $X$ and $Y$ are fixed,

$$
E(X Y) \leq \int x \Phi^{-1}(P(x)) p(x) \mathrm{d} x .
$$

If we now take $P(x)=\Phi((x-\mu) / \sigma)$ then

$$
\int x \Phi^{-1}(P(x)) p(x) \mathrm{d} x=E\left[X\left(\frac{X-\mu)}{\sigma}\right)\right]=\sigma .
$$

Hence, (6) is a sharp upper bound with equality when $X$ is also normal. 
To see how extensive the improved inequality is, let us consider a simple example with $Y$ as standard normal and $X$ has mean $\mu$ and variance $\sigma^{2}$. Then the Cauchy-Schwarz inequality gives $[E(X Y)]^{2} \leq \mu^{2}+\sigma^{2}$, whereas the improved inequlity yields $[E(X Y)]^{2} \leq \sigma^{2}$, which clearly can amount to a large difference.

\subsection{General Cramer-Rao inequality}

Consider a regular parametric model $p_{\theta}(\cdot)$ and an observation $x$ from the model. Then for an unbiased estimator $\widehat{\theta}$ for $\theta$ we have the well known Cramer-Rao lower bound for the variance of $\widehat{\theta}$; namely $\operatorname{Var}(\widehat{\theta}) \geq 1 / I(\theta)$ where $I(\theta)$ is the Fisher information, given by

$$
I(\theta)=\int\left(\frac{\partial}{\partial \theta} \log p_{\theta}(x)\right)^{2} p_{\theta}(x) \mathrm{d} x .
$$

Here we provide a lower bound for mean square error when the estimator is biased.

Let $T$ be a biased estimator for $\theta$; so $E(T)=\theta+b(\theta)$. Now using the extended Cauchy-Schwarz inequality (5), we have

$$
\left[\int(T-\theta) Y p_{\theta}(x) \mathrm{d} x\right]^{2} \leq E\left[(T-\theta)^{2}\right] E\left(Y^{2}\right)-[E(T-\theta)]^{2} \operatorname{Var}(Y),
$$

where

$$
Y=\frac{\partial}{\partial \theta} \log p_{\theta}(X)
$$

and so $E(Y)=0$ and $E\left(Y^{2}\right)=\operatorname{Var}(Y)=I(\theta)$. The term on the left hand side of (7) is, allowing the switch $\int \partial / \partial \theta \equiv \partial / \partial \theta \int$, equal to $\left(1+b^{\prime}(\theta)\right)^{2}$ and hence

$$
\mathrm{MSE}=E\left[(T-\theta)^{2}\right] \geq \frac{\left(1+b^{\prime}(\theta)\right)^{2}}{I(\theta)}+b^{2}(\theta) .
$$

This is a sharp lower bound for estimators of the form $T=w \widehat{\theta}+(1-w) \mu$, where $\widehat{\theta}$ is unbiased and has variance $1 / I(\theta)$. For with this form, a Bayes estimator for example, we have

$$
(T-\theta)^{2}=[w(\widehat{\theta}-\theta)+(1-w)(\mu-\theta)]^{2} .
$$


Hence,

$$
E\left[(T-\theta)^{2}\right]=\frac{w^{2}}{I(\theta)}+(1-w)^{2}(\mu-\theta)^{2} .
$$

Now note that $b(\theta)=(1-w)(\mu-\theta)$ and $b^{\prime}(\theta)=w-1$. Clearly, we also have that for biased estimator $T, \operatorname{Var}(T) \geq\left(1+b^{\prime}(\theta)\right)^{2} / I(\theta)$, where bias $(T)=b(\theta)$.

\section{Acknowledgement}

The authors's work is partially supported by NSF grant: DMS Award 1506879 .

\section{References}

[1] J. M. Aldaz, Strengthened Cauchy-Schwarz and Hölder inequalities, J. Inequal. Pure Appl. Math 10 (2009), art. 116.

[2] H. Alzer, A refinement of the Cauchy-Schwarz inequality, J. Math. Anal. Appl. 168 (1992), 596-604.

[3] S. G. Bobkov, N. Gozlan, C. Roberto and P. M. Samson, Bounds on the deficit in the logarithmic Sobolev inequality, J. Funct. Anal. 267 (2014), 4110-4138.

[4] S. S. Dragomir, A survey on Cauchy-Buntakowsky-Schwarz type discrete inequalities, J. Inequal. Pure Appl. Math. 4 (2003), art. 63.

[5] F. MÉmoli, The Gromov-Wasserstein distance: A brief overview, Axioms 3 (2014), 335-341.

[6] J. M. Steele, The Cauchy-Schwarz Master Class, (2004), Cambridge University Press, NY.

[7] L. TuO, Generalizations of Cauchy-Schwarz inequality in unitary spaces, J. Inequal. Appl. (2015), art. 201.

[8] S. G. WALkER, On a lower bound for the Jensen inequality, SIAM J. Math. Anal. 46 (2014), 3151-3157. 